\title{
RESEARCH
}

Open Access

\section{Iron homeostasis pathway DNA methylation trajectories reveal a role for STEAP3 metalloreductase in patient outcomes after aneurysmal subarachnoid hemorrhage}

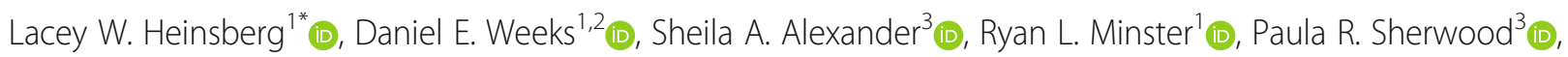
Samuel M. Poloyac ${ }^{4} \mathbb{D}$, Sandra Deslouches ${ }^{1,5} \mathbb{D}$, Elizabeth A. Crago ${ }^{3}$ (D) and Yvette P. Conley ${ }^{1,5}$ (D)

\begin{abstract}
Background: Following aneurysmal subarachnoid hemorrhage (aSAH), the brain is susceptible to ferroptosis, a type of iron-dependent cell death. Therapeutic intervention targeting the iron homeostasis pathway shows promise for mitigating ferroptosis and improving recovery in animal models, but little work has been conducted in humans. DNA methylation (DNAm) plays a key role in gene expression and brain function, plasticity, and injury recovery, making it a potentially useful biomarker of outcomes or therapeutic target for intervention. Therefore, in this longitudinal, observational study, we examined the relationships between trajectories of DNAm in candidate genes related to iron homeostasis and acute (cerebral vasospasm and delayed cerebral ischemia) and long-term (Glasgow Outcome Scale [GOS, unfavorable $=1-3]$ and death) patient outcomes after aSAH.
\end{abstract}

\footnotetext{
*Correspondence: law145@pitt.edu

1 Department of Human Genetics, Graduate School of Public Health, University of Pittsburgh, Pittsburgh, PA 15261, USA

Full list of author information is available at the end of the article
}

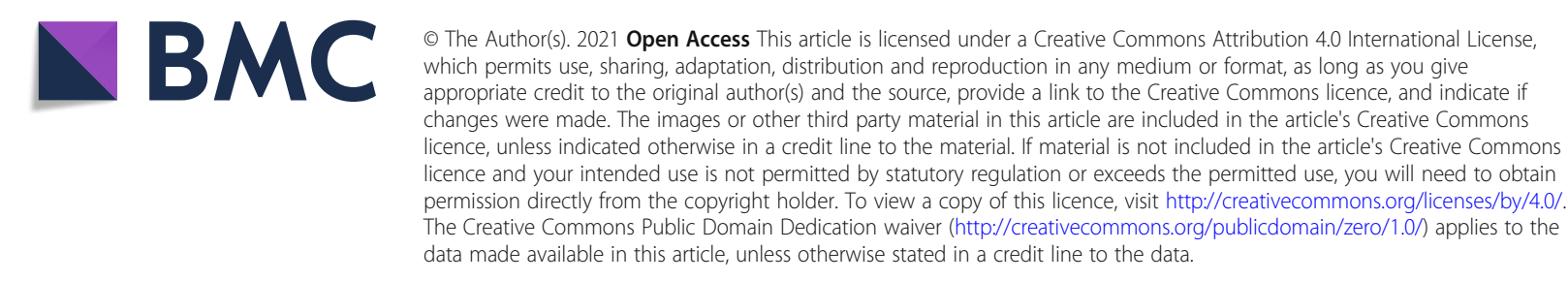


Results: Longitudinal, genome-wide DNAm data were generated from DNA extracted from post-aSAH cerebrospinal fluid ( $n=260$ participants). DNAm trajectories of $637 \mathrm{CpG}$ sites in 36 candidate genes related to iron homeostasis were characterized over 13 days post-aSAH using group-based trajectory analysis, an unsupervised clustering method. Significant associations were identified between inferred DNAm trajectory groups at several CpG sites and acute and long-term outcomes. Among our results, cg25713625 in the STEAP3 metalloreductase gene (STEAP3) stood out. Specifically, in comparing the highest cg25713625 DNAm trajectory group with the lowest, we observed significant associations (i.e., based on $p$-values less than an empirical significance threshold) with unfavorable GOS at 3 and 12 months ( $O R=11.7, p=0.0006$ and $O R=15.6, p=0.0018$, respectively) and death at 3 and 12 months $(O R=19.1, p=0.0093$ and $O R=12.8, p=0.0041$, respectively). These results were replicated in an independent sample ( $n=100$ participants) observing significant associations with GOS at 3 and 12 months (OR= 8.2, $p=0.001$ and $O R=6.3, p=0.0 .0047$, respectively) and death at 3 months $(O R=2.3, p=0.008)$ and a suggestive association (i.e., $p$-value $<0.05$ not meeting an empirical significance threshold) with death at 12 months $(O R=2.0$, $p=0.0272$ ). In both samples, an additive effect of the DNAm trajectory group was observed as the percentage of participants with unfavorable long-term outcomes increased substantially with higher DNAm trajectory groups.

Conclusion: Our results support a role for DNAm of cg25713625/STEAP3 in recovery following aSAH. Additional research is needed to further explore the role of DNAm of cg25713625/STEAP3 as a biomarker of unfavorable outcomes, or therapeutic target to improve outcomes, to translate these findings clinically.

Keywords: Epigenetics, Biomarker, Group-based trajectory analysis, Six-transmembrane epithelial antigen of prostate 3, Stroke

\section{Background}

Aneurysmal subarachnoid hemorrhage (aSAH) is a devastating type of stroke with substantial variability in patient recovery and outcomes. The 30-day fatality rate falls between 25 and $50 \%$ [1,2], and approximately $60 \%$ of those who survive experience a range of symptoms and disability that can affect the ability to perform essential functions such as activities of daily living, returning to work, and maintaining healthy relationships [3, 4]. Poor outcomes post-hospitalization have been linked to brain injury that occurs during the first 2 weeks postaSAH, including acute complications such as cerebral vasospasm $(\mathrm{CV})$ and delayed cerebral ischemia (DCI) [1]. Despite the strong association between CV, DCI, and long-term functional outcomes, the pathophysiology of these complications remains largely unknown and interventions targeting early brain injury have not been successful in improving long-term outcomes post-aSAH $[5]$.

Iron and related homeostatic mechanisms are potentially important factors in response to aSAH [6-11]. Normally, iron is tightly bound to carrier proteins as ferric iron [12]. However, after aSAH, blood contaminates the subarachnoid space and cerebrospinal fluid (CSF) where heme is broken down into carbon monoxide, biliverdin, and non-protein-bound ferrous iron (i.e., free iron) [13]. "Ferroptosis" is a unique type of irondependent cell death that results in secondary ischemic brain injury following experimental subarachnoid hemorrhage $[14,15]$. Iron chelators effectively reduce associated neuronal cell death in animal models of neurologic injury [15-18], and preliminary research in humans supports the potential importance of iron homeostasis in recovery from aSAH $[6,7,10,19]$.

DNA methylation (DNAm) plays a key role in gene expression and has a significant impact on adult brain function, plasticity, and injury recovery [20], making it a potentially useful biomarker of outcomes or therapeutic target for intervention to improve outcomes after aSAH. While little DNAm research has been performed in the aSAH population specifically, peripheral blood DNA hypomethylation of a candidate region of the genome has been associated with increased mortality after ischemic stroke [21] and global DNA hypomethylation in damaged brain tissue has been observed post-injury in rats after traumatic brain injury [22]. Because DNAm is tissue- and time-sensitive, examining DNAm in CSF acutely post-aSAH may uncover important evidence about the pathophysiology of patient recovery and unfavorable outcomes as the CSF clears, particularly in the context of genes critical to iron homeostasis.

Previously, we developed an objective protocol to largely automate group-based trajectory analysis (GBTA) of longitudinal CSF DNAm data using an exemplar "master" iron regulatory gene, hepcidin (HAMP) [9]. GBTA, an unsupervised clustering method, is an ideal approach for clinical examination of DNAm data as it allows one to test if the sample is composed of distinct groups, each with a different underlying trajectory. The identification of DNAm sites that vary over time - and are associated with later outcomes after aSAH - may be useful in identifying subpopulations of patients that 
require more intensive clinical management to facilitate optimal stroke care delivery and improve patient outcomes. In addition to understanding associations with acute outcomes, it is important to know if CSF DNAm trajectories are associated with long-term outcomes either directly or indirectly via an unmeasured acute complication of vascular or microvascular dysfunction. Therefore, the purpose of this study was to investigate associations between inferred DNAm trajectory groups in candidate genes related to iron homeostasis and acute and long-term patient outcomes following aSAH.

\section{Results}

\section{Sample characteristics and overview of study workflow and findings}

As shown in the Fig. 1 study overview and workflow, our final discovery and replication samples consisted of 260 and 100 participants, respectively, with longitudinal DNAm data. Thirty-nine candidate genes were selected based on their known biological roles in iron homeostasis and are listed in the Supplementary Information (SI, Table S1) and described in detail as part of our previous, related work $[9,10]$.

Sample characteristics are presented in Table 1. Primary acute outcomes of interest included $\mathrm{CV}$ and $\mathrm{DCI}$ (occurring during the first 2 weeks post-aSAH), and long-term outcomes included Glasgow Outcome Scale (GOS, unfavorable $=1-3)$ and death at 3 and 12 months post-aSAH. Longitudinal CSF DNAm data were available for participants over 13 and 14 days post-injury in the discovery and replication analyses, respectively, as detailed in the methods. Participants had between 2 and 5 DNAm observations with an average of 3.2 observations in our discovery sample and between 2 and 4 DNAm observations with an average of 3.0 observations in our replication sample. Details of the number of samples available per day are presented in the SI (Table S2 and Fig. S2).

In the entire aSAH cohort $(N=648$ participants $)$, older age was associated with DCI occurrence $(p=0.04)$,

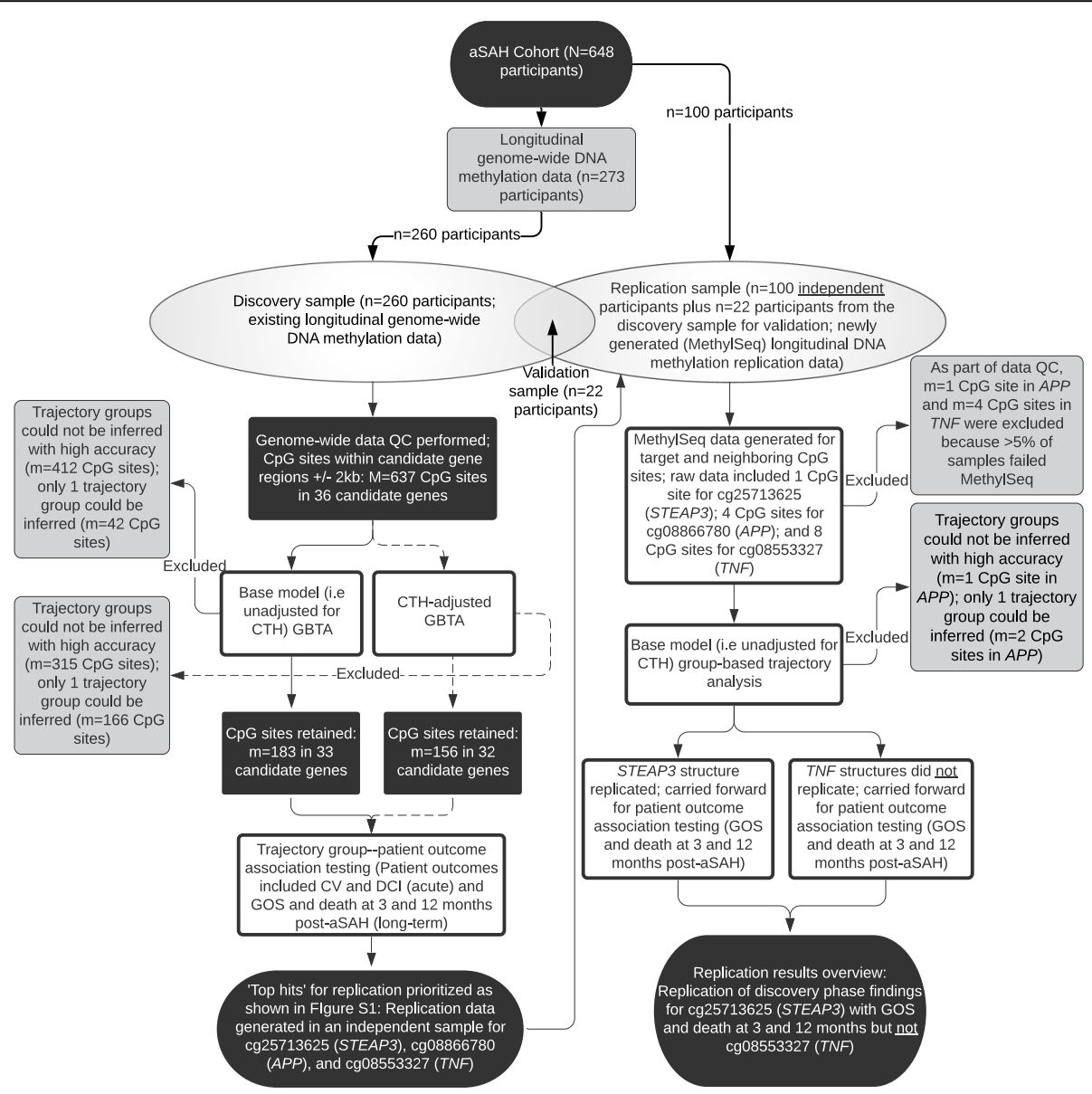

Fig. 1 Overview of study workflow and findings. aSAH, aneurysmal subarachnoid hemorrhage; QC, quality control; GBTA, group-based trajectory analysis; CTH, cell type heterogeneity; CV, cerebral vasospasm; DCl, delayed cerebral ischemia; GOS, Glasgow Outcome Scale; N/n notation refers to the number of participants; $M / m$ notation refers to the number of $\mathrm{CpG}$ sites; dashed lines represent $\mathrm{CTH}$-adjusted analyses, which were considered a secondary focus of this study given our search for a clinical biomarker; candidate genes correspond to those shown in Table S1 
Table 1 Participant characteristics for the entire aneurysmal subarachnoid hemorrhage cohort and discovery and replication sample subsets

\begin{tabular}{|c|c|c|c|c|c|c|}
\hline \multirow{2}{*}{$\begin{array}{l}\text { Variable } \\
\text { Age, mean years }(S D)\end{array}$} & \multicolumn{2}{|c|}{ aSAH cohort $(n=648)$} & \multicolumn{2}{|c|}{ Discovery sample $(n=260)$} & \multicolumn{2}{|c|}{ Replication sample $(n=100)$} \\
\hline & \multicolumn{2}{|c|}{$53.2(11.5)$} & \multicolumn{2}{|c|}{$53.1(11.0)$} & \multicolumn{2}{|c|}{$54.2(12.0)$} \\
\hline Sex, female, $n(\%)$ & \multicolumn{2}{|c|}{$469(72.4)$} & \multicolumn{2}{|c|}{$179(68.8)$} & \multicolumn{2}{|c|}{$76(76.0)$} \\
\hline Self-identified race, white, $n$ (\%) & \multicolumn{2}{|c|}{$563(86.9)$} & \multicolumn{2}{|c|}{$225(86.5)$} & \multicolumn{2}{|c|}{$91(91.0)$} \\
\hline Treatment, coil embolization, $n(\%)$ & \multicolumn{2}{|c|}{$402(62.0)$} & \multicolumn{2}{|c|}{$159(61.2)$} & \multicolumn{2}{|c|}{$66(66.0)$} \\
\hline \multicolumn{7}{|l|}{ Fisher grade, $n(\%)$} \\
\hline 2 & \multicolumn{2}{|c|}{$266(41.0)$} & \multicolumn{2}{|c|}{$78(30.0)$} & \multicolumn{2}{|c|}{$41(41.0)$} \\
\hline 3 & \multicolumn{2}{|c|}{$279(43.1)$} & \multicolumn{2}{|c|}{$126(48.5)$} & \multicolumn{2}{|c|}{$43(43.0)$} \\
\hline 4 & \multicolumn{2}{|c|}{$103(15.9)$} & \multicolumn{2}{|c|}{$56(21.5)$} & \multicolumn{2}{|c|}{$16(16.0)$} \\
\hline Outcome & $N$ & Unfavorable, n (\%) & N & Unfavorable, $n(\%)$ & $N$ & Unfavorable, $n(\%)$ \\
\hline CV & 396 & $206(52.0)$ & 168 & $91(54.2)$ & \multicolumn{2}{|c|}{$N A^{a}$} \\
\hline $\mathrm{DCl}$ & 631 & $253(40.1)$ & 258 & $127(49.2)$ & & \\
\hline GOS-3 & 555 & $149(26.8)$ & 214 & $71(33.2)$ & 99 & $38(38.4)$ \\
\hline GOS-12 & 530 & $121(22.8)$ & 204 & $53(26.0)$ & 93 & $29(31.2)$ \\
\hline Death-3 & 594 & 88 (14.8) & 232 & 39 (16.8) & 99 & $24(24.2)$ \\
\hline Death-12 & 530 & $96(18.1)$ & 204 & $44(21.6)$ & 93 & $26(28.0)$ \\
\hline
\end{tabular}

$a S A H$, aneurysmal subarachnoid hemorrhage; $S D$, standard deviation; $C V$, cerebral vasospasm (unfavorable $=C V$ present); $D C l$, delayed cerebral ischemia (unfavorable = DCI present); GOS-3, Glasgow Outcome Scale at 3 months (unfavorable = 1-3); GOS-12, Glasgow Outcome Scale at 12 months (unfavorable = 1-3); Death-3, death at 3 months (unfavorable = yes); Death-12, death at 12 months (unfavorable = yes); ${ }^{\text {a }} \mathrm{CV}$ and DCl were not examined in the replication sample given null association in discovery analyses

unfavorable GOS at 3 and 12 months $(p=0.03$ and $p=$ 0.04 , respectively), and death at 3 and 12 months ( $p=$ 0.02 and $p=0.04)$. Self-reported race was associated with GOS at 3 months $(p=0.04)$, with individuals who self-identified as White having a higher percentage of favorable GOS scores. Higher Fisher grade was associated with the occurrence of CV $(p=0.01)$ and DCI $(p=$ 0.03 ), as well as unfavorable GOS and death measured at 3 and 12 months (all $p<0.001$ ). As such, age, selfreported race, and Fisher grade were included as covariates in our logistic regression models. No significant associations between sex (assigned at birth) and patient outcomes or intervention (surgical vs. coil embolization) and patient outcomes were observed. However, sex was included in our models given the importance of estrogen response elements in iron homeostasis [23].

From the genome-wide data, DNAm data for 36 candidate genes and $637 \mathrm{CpG}$ sites were available and analyzed as part of the targeted discovery phase (Table S1). During data screening, DNAm observations that were identified as extreme outliers $>3$ times the interquartile range were score adjusted to prevent bias in GBTA (Table S1) as justified in the SI. As detailed in the methods, to both evaluate the clinical utility of our work and aid in biological interpretation, we performed GBTA both with and without adjusting for cell type heterogeneity $(\mathrm{CTH})$. For our base modeling (i.e., unadjusted for $\mathrm{CTH}$ ), which was the primary focus of this study given the increased likelihood of clinical utility, our objective protocol eliminated $412 \mathrm{CpG}$ sites as trajectory groups could not be inferred with high accuracy. At an additional 42 CpG sites, we inferred only one trajectory group. After elimination of these sites from our analysis, $183 \mathrm{CpG}$ sites in 33 of our candidate genes were carried forward for patient outcome association testing (Fig. 1). A summary of our CTH-adjusted workflow is provided in parallel (Fig. 1). In examining associations between site-specific inferred DNAm trajectory groups and patient outcomes, we reported unadjusted $p$-values throughout, but judged significance based on empirical thresholds as detailed in the methods. An overview of the results of associations with a $p$-value $<0.05$ are presented in the SI for base models (Table S3) and CTHadjusted models (Table S4) and are detailed in genespecific tables (Additional File 2).

Three CpG sites in three candidate genes were flagged as noteworthy of replication as outlined in the SI (Fig. S1 and Table S5). These sites included cg25713625 in STEAP3 metalloreductase (STEAP3), cg08866780 in the amyloid precursor protein (APP), and cg08553327 in tumor necrosis factor $(T N F)$. Given the breadth of this study, we discuss the discovery and replication results for only these three CpG sites, but results of all discovery analyses are presented in the SI as described above. Discovery phase trajectory plots for our top three hits in STEAP3, APP, and TNF are presented in Fig. 2 and in the SI (Fig. S2 and Table S6). Discovery phase participant characteristics by trajectory group are presented in 


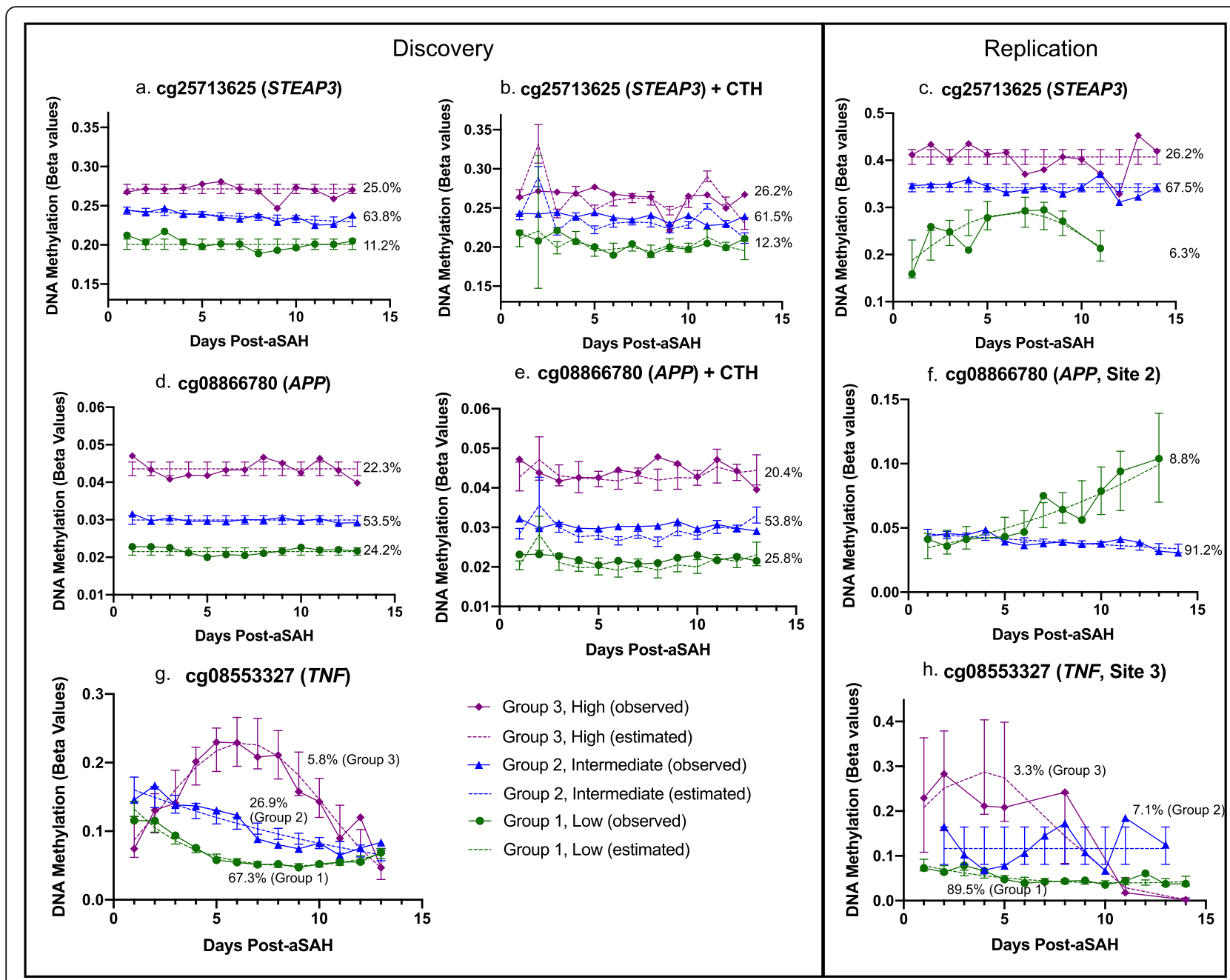

Fig. 2 Discovery and replication DNA methylation trajectory plots for top hits. Inferred trajectory groups computed using group-based trajectory analysis separated by discovery phase (base models and CTH-adjusted models) and replication phase (base models only) with group membership percentages depicted; $y$-axis scale, 0 to 1; the CTH-adjusted trajectory model for cg08553327 (TNF) did not pass posterior model quality control and therefore is not included here; bars plot the $95 \%$ confidence interval for the estimate; DNA methylation data were analyzed as $M$ values and converted to beta values for plot presented here; a table with exact DNA methylation levels as measured by beta values is presented in Table S6

the SI (Tables S7 and S8). It should be noted, however, that the base trajectory models are not directly comparable to the $\mathrm{CTH}$-adjusted trajectory models as group membership changes slightly between the analyses (Fig. S3). Discovery phase binary logistic regression results exploring associations of inferred DNAm trajectory groups with patient outcomes are presented for our top hits in Table 2.

As described below, probe sequences for replication by pyrosequencing (i.e., MethylSeq) were designed to capture top hits and regions surrounding top hits. For $\operatorname{cg} 08866780(A P P)$ and cg08553327 (TNF), this captured the target $\mathrm{CpG}$ sites and additional variable CpG sites in this region for a total of 4 sites $(A P P)$ and 8 sites $(T N F)$. After replication data quality control (QC), for our top hits of interest in STEAP3,
$A P P$, and $T N F$, we examined a total of 1,3 , and 4 replication sites, respectively.

\section{Detailed discovery and replication results cg25713625 (STEAP3)}

For cg25713625 (STEAP3), in the discovery and replication base models (i.e., unadjusted for CTH, Fig. 2a and c, respectively), we inferred three trajectory groups with similar group membership percentages including a low DNAm group (group 1, 11.2\% [discovery], 6.3\% [replication]), intermediate DNAm group (group 2, 63.8\% [discovery], 67.5\% [replication]), and high DNAm group (group 3, 25.0\% [discovery], 26.2\% [replication]). Trajectory shapes appeared similar between the discovery and replication samples, though the low DNAm group had a quadratic shape during replication. After controlling for 
Table 2 Discovery results of binary logistic regression and global analysis examining associations of cg25713625 (STEAP3), cg08866780 (APP), and cg08553327 (TNF) unadjusted and CTH-adjusted trajectory groups with patient outcomes while controlling for age, sex, self-identified race, and Fisher grade

\section{Table 2a. cg25713625 (STEAP3)}

Base model, polynomial order 010, Fig. 2a

Group 2 (intermediate) vs. group 1 (low)

Group 3 (high) vs. group 1 (low)

\begin{tabular}{|c|c|c|c|c|c|c|c|}
\hline Outcome & $O R$ & $95 \% \mathrm{Cl}$ & $p^{c}$ & $O R$ & $95 \% \mathrm{Cl}$ & $p^{c}$ & Global $p^{\mathrm{d}}$ \\
\hline CV & 0.662 & 0.21 to 2.03 & 0.471 & 1.126 & 0.33 to 3.80 & 0.847 & 0.351 \\
\hline $\mathrm{DCl}$ & 1.032 & 0.45 to 2.41 & 0.941 & 1.256 & 0.49 to 3.20 & 0.629 & 0.789 \\
\hline GOS-3 & 2.982 & 0.86 to 12.56 & 0.1 & 11.743 & 3.14 to 21.65 & $0.0006^{a}$ & $0.00005^{a}$ \\
\hline GOS-12 & 6.346 & 1.47 to 31.32 & $0.029^{b}$ & 15.595 & 3.35 to 22.54 & $0.0018^{\mathrm{a}}$ & $0.0005^{a}$ \\
\hline Death-3 & 7.147 & 1.00 to 32.53 & 0.076 & 19.081 & 3.01 to 25.12 & $0.0093^{\mathrm{b}}$ & $0.0013^{a}$ \\
\hline Death-12 & 4.859 & 1.00 to 35.80 & 0.064 & 12.762 & 2.71 to 25.13 & $0.0041^{b}$ & $0.0015^{a}$ \\
\hline \multicolumn{8}{|c|}{$\mathrm{CTH}$-adjusted model, polynomial order 000, Fig. 2b } \\
\hline & \multicolumn{3}{|c|}{ Group 2 (intermediate) vs. group 1 (low) } & \multicolumn{3}{|c|}{ Group 3 (high) vs. group 1 (low) } & \\
\hline Outcome & $O R$ & $95 \% \mathrm{Cl}$ & $p^{c}$ & $O R$ & $95 \% \mathrm{Cl}$ & $p^{c}$ & Global $p^{\mathrm{d}}$ \\
\hline CV & 0.433 & 0.14 to 1.26 & 0.131 & 0.832 & 0.25 to 2.70 & 0.76 & 0.129 \\
\hline $\mathrm{DCl}$ & 0.612 & 0.27 to 1.36 & 0.231 & 0.913 & 0.37 to 2.20 & 0.84 & 0.260 \\
\hline GOS-3 & 2.589 & 0.80 to 9.35 & 0.115 & 4.143 & 1.25 to 5.01 & $\underline{0.0271^{\mathrm{b}}}$ & 0.062 \\
\hline GOS-12 & 3.960 & 1.00 to 10.58 & 0.055 & 5.972 & 1.52 to 6.25 & $0.0182^{\mathrm{b}}$ & $0.0326^{\mathrm{b}}$ \\
\hline Death-3 & 3.768 & 0.90 to 11.54 & 0.108 & 4.847 & 1.05 to 7.50 & 0.069 & 0.122 \\
\hline Death-12 & 3.120 & 0.86 to 15.63 & 0.115 & 4.249 & 1.06 to 5.25 & 0.059 & 0.123 \\
\hline
\end{tabular}

Table 2b. cg08866780 (APP)

Base model, polynomial order 000, Fig. 2d

Group 2 (intermediate) vs. group 1 (low)

$\begin{array}{lll}\text { Outcome } & \text { OR } & 95 \% \text { Cl } \\ \text { CV } & 0.443 & 0.19 \text { to } 0.989 \\ \text { DCl } & 0.803 & 0.44 \text { to } 1.48 \\ \text { GOS-3 } & 0.349 & 0.16 \text { to } 0.74 \\ \text { GOS-12 } & 0.194 & 0.08 \text { to } 0.44 \\ \text { Death-3 } & 0.209 & 0.08 \text { to } 0.52 \\ \text { Death-12 } & 0.206 & 0.08 \text { to } 0.50\end{array}$

Group 3 (high) vs. group 1 (low)

OR $\quad 95 \% \mathrm{Cl}$

$p^{c}$

0.051

0.366

0.14 to 1.00

0.56 to 2.41

0.479

1.156

0.32 to 1.82

0.39 to 1.98

0.44 to 2.67

0.39 to 2.43

0.977

0.826

$0.0010^{\mathrm{a}}$

$0.0006^{\mathrm{a}}$

CTH-adjusted model, polynomial order 000, Fig. 2e

Group 2 (intermediate) vs. group 1 (low)

$\begin{array}{llll}\text { Outcome } & \text { OR } & 95 \% \mathrm{Cl} & p^{c} \\ \text { CV } & 0.813 & 0.37 \text { to } 1.76 & 0.602 \\ \text { DCl } & 1.230 & 0.68 \text { to } 2.24 & 0.494 \\ \text { GOS-3 } & 0.332 & 0.16 \text { to } 0.70 & \underline{0.0040^{b}} \\ \text { GOS-12 } & 0.347 & 0.15 \text { to } 0.77 & \underline{\underline{0.0097^{b}}} \\ \text { Death-3 } & 0.359 & 0.15 \text { to } 0.87 & \underline{\underline{0.0237^{b}}} \\ \text { Death-12 } & 0.337 & 0.14 \text { to } 0.79 & \underline{\underline{0.0128}}\end{array}$

Group 3 (high) vs. group 1 (low)

OR

0.654

1.400

0.683

1.070

1.284

1.143

\begin{abstract}
$95 \% \mathrm{Cl}$
0.25 to 1.67

0.67 to 2.97

0.27 to 1.67

0.43 to 2.68

0.50 to 3.31

0.44 to 2.95
\end{abstract}

$p^{c}$

0.056

0.698

0.541

0.672

0.868

0.96

$p^{c}$

0.374

0.377

0.4072

0.8853

0.6028

0.7817
Global $p^{\mathrm{d}}$

0.069

0.488

$\underline{0.0118^{\mathrm{b}}}$

$0.0001^{a}$

$0.0002^{\mathrm{a}}$

$0.0002^{a}$ 
Table 2 Discovery results of binary logistic regression and global analysis examining associations of cg25713625 (STEAP3), cg08866780 (APP), and cg08553327 (TNF) unadjusted and CTH-adjusted trajectory groups with patient outcomes while controlling for age, sex, self-identified race, and Fisher grade (Continued)

Table 2c. cg08553327 (TNF)

Base model, polynomial order 212, Fig. $2 \mathrm{~g}$

Group 2 (intermediate) vs. group 1 (low)

\begin{tabular}{llll} 
Outcome & OR & $95 \% \mathrm{Cl}$ & $p^{c}$ \\
CV & 0.850 & 0.42 to 1.72 & 0.651 \\
DCl & 0.827 & 0.47 to 1.46 & 0.516 \\
GOS-3 & 3.686 & 1.78 to 7.87 & $\mathbf{0 . 0 0 0 5}$ \\
GOS-12 & 2.080 & 0.98 to 4.43 & 0.0568 \\
Death-3 & 2.488 & 1.11 to 5.57 & $\underline{0.0257}$ \\
Death-12 & 2.496 & 1.12 to 5.56 & $\underline{0.0243}$ \\
\hline
\end{tabular}

Group 3 (high) vs. group 1 (low)

$O R$

1.223

3.066

13.134

4.488

5.603

7.123
95\% Cl

0.31 to 5.36

0.98 to 11.64

3.51 to 39.02

1.24 to 16.50

1.47 to 20.44

1.89 to 27.94 $p^{c}$

0.777

0.069

$\mathbf{0 . 0 0 0 3 ^ { a }}$

$0.0209^{\mathrm{b}}$

$0.0089^{\mathrm{b}}$

$0.0037^{b}$
Global $p^{\mathrm{d}}$

0.844

0.101

$0.00001^{2}$

$0.0244^{b}$

$0.0094^{b}$

$0.0038^{\mathrm{b}}$

Corresponds to trajectory groups depictured in Fig. 2a (STEAP3), 2b (STEAP3 + CTH), 2d (APP), 2e (APP + CTH), and 2g (TNF). Base model, unadjusted for CTH; OR, odds ratio; $C l$, confidence interval; $p, p$-value based on an alpha of 0.05 ; Global $p, p$-value computed using partial $F$ test comparing models with and without trajectory group; $C T H$, cell type heterogeneity; $C V$, cerebral vasospasm; $D C l$, delayed cerebral ischemia; GOS-3, Glasgow Outcome Scale at 3 months (unfavorable = 1-3); GOS-12, Glasgow Outcome Scale at 12 months (unfavorable = 1-3); Death-3, death at 3 months; Death-12, death at 12 months. ${ }^{a}$ Significant associations (meeting empirical significance level) have been bolded. ${ }^{b}$ Suggestive associations (unadjusted $p<0.05$ ) have been underlined. ${ }^{c}$ Empirical significance threshold $=$ 0.002 (calculated based on the minimum of $12 p$-values, including both group 2 vs. group 1 and group 3 vs. group 1 comparisons, in permutation testing).

${ }^{\mathrm{d}}$ Empirical significance threshold for global analysis $=0.003$ (calculated based on the minimum of $6 p$-values in permutation testing)

$\mathrm{CTH}$ in the discovery phase (Fig. 2b), we observed nearly identical trajectories and group membership percentages to those observed in the base model. Participant characteristics by base model trajectory groups are presented for the discovery and replication phase in Table 3 and by CTH-adjusted model trajectory groups for the discovery phase in the SI (Tables S7 and S8). As shown in Table 3, in the discovery phase, the low DNAm group had fewer female participants $(p=0.011)$ and individuals who self-reported their race as white $(p=0.013)$ while in the replication phase, the low DNAm group had a higher mean age $(p=0.03)$. No other participant differences by trajectory group were observed.
In the discovery phase association analyses (Table 2a), significant associations (i.e., $p$-value less than the empirical significance threshold computed in permutation testing) were identified with unfavorable GOS at 3 and 12 months $(p=0.0006$ and $p=0.002$, respectively) and suggestive associations (i.e., $p$-value $<0.05$ not meeting the empirical significance threshold) were identified with death at 3 and 12 months $(p=0.009$ and $p=0.004$, respectively). Overall, we observed an increased odds of unfavorable long-term outcomes $(O R>11$ for all $)$ in the high DNAm group compared with the low DNAm group. In our CTH-adjusted models, suggestive associations were again observed with GOS at 3 and 12 months $(p=0.03$ and $p=0.02$, respectively). No significant

Table 3 Participant characteristics by trajectory groups for cg25713625 (STEAP3) discovery and replication samples

\begin{tabular}{|c|c|c|c|c|c|c|c|c|}
\hline \multirow[b]{2}{*}{ Variable } & \multicolumn{4}{|c|}{ Discovery $(n=260)$} & \multicolumn{4}{|c|}{ Replication $(n=100)$} \\
\hline & $\begin{array}{l}\text { Group } 1 \\
\text { (low) }\end{array}$ & $\begin{array}{l}\text { Group } 2 \\
\text { (intermediate) }\end{array}$ & $\begin{array}{l}\text { Group } 3 \\
\text { (high) }\end{array}$ & $p$ & $\begin{array}{l}\text { Group } 1 \\
\text { (low) }\end{array}$ & $\begin{array}{l}\text { Group } 2 \\
\text { (intermediate) }\end{array}$ & $\begin{array}{l}\text { Group } 3 \\
\text { (high) }\end{array}$ & $p$ \\
\hline Group membership, $n$ (\%) & $29(11.1)$ & $166(63.8)$ & $65(25.0)$ & & $6(6.0)$ & $68(68.0)$ & $26(26.0)$ & \\
\hline Age, mean (SD) & $52.3(12.2)$ & $53.0(10.8)$ & $53.6(11.2)$ & $0.862^{\mathrm{a}}$ & $65.2(6.6)$ & $54.4(12.0)$ & $51.0(11.6)$ & $0.03^{a}$ \\
\hline Sex, female, $n(\%)$ & $13(44.8)$ & $118(71.1)$ & $48(73.8)$ & $0.011^{b}$ & $4(66.7)$ & $53(77.9)$ & 19 (73.1) & $0.667^{c}$ \\
\hline $\begin{array}{l}\text { Self-identified race, white, } \\
n(\%)\end{array}$ & $20(69.0)$ & 147 (88.6) & $58(89.2)$ & $0.013^{b}$ & $6(100)$ & $61(89.7)$ & $24(92.3)$ & $1^{c}$ \\
\hline \multicolumn{9}{|l|}{ Fisher grade } \\
\hline 2 & $6(20.7)$ & $54(32.5)$ & $18(27.7)$ & $0.558^{b}$ & $5(83.3)$ & $27(39.7)$ & $9(34.6)$ & $0.719^{b, d}$ \\
\hline 3 & $14(48.3)$ & $80(48.2)$ & $32(49.2)$ & & $1(16.7)$ & $30(44.1)$ & $12(46.2)$ & \\
\hline 4 & $9(31.0)$ & $32(19.3)$ & $15(23.1)$ & & 0 & $11(16.2)$ & $5(19.2)$ & \\
\hline Intervention, coil, n (\%) & $16(55.2)$ & 99 (59.6) & $40(61.5)$ & $0.845^{b}$ & $4(66.7)$ & $46(67.6)$ & $16(61.5)$ & $0.875^{c}$ \\
\hline
\end{tabular}

Discovery, trajectory groups depicted in Fig. 2a; Replication, trajectory groups depicted in Fig. 2c; SD, standard deviation; significant associations based on $p<0.05$ have been bolded. a One-way analysis of variance; ${ }^{b}$ chi-square test of independence; ${ }^{~}$ Fisher's exact test; ${ }^{d}$ computed by combining low and intermediate groups because of 0 cell count 
Table 4 Outcome distributions by trajectory group in cg25713625 (STEAP3) discovery and replication samples

\begin{tabular}{|c|c|c|c|c|c|c|}
\hline \multirow[b]{2}{*}{ Group } & \multicolumn{3}{|c|}{ Discovery $(n=214)$} & \multicolumn{3}{|c|}{ Replication $(n=99)$} \\
\hline & Favorable, $n$ & Unfavorable, $n$ & $\%$ Unfavorable & Favorable, $n$ & Unfavorable, $n$ & $\%$ Unfavorable \\
\hline 3 (high) & 23 & 29 & 55.77 & 10 & 16 & 61.54 \\
\hline 2 (intermediate) & 101 & 38 & 27.34 & 45 & 22 & 32.84 \\
\hline 1 (low) & 19 & 4 & 17.39 & 6 & 0 & 0.00 \\
\hline \multicolumn{7}{|l|}{ GOS-12 } \\
\hline & \multicolumn{3}{|c|}{ Discovery $(n=204)$} & \multicolumn{3}{|c|}{ Replication $(n=93)$} \\
\hline Group & Favorable, $n$ & Unfavorable, $n$ & $\%$ Unfavorable & Favorable, $n$ & Unfavorable, $n$ & \% Unfavorable \\
\hline 3 (high) & 32 & 22 & 40.74 & 12 & 13 & 52.00 \\
\hline 2 (intermediate) & 97 & 29 & 23.02 & 46 & 16 & 25.81 \\
\hline 1 (low) & 22 & 2 & 8.33 & 6 & 0 & 0.00 \\
\hline \multicolumn{7}{|l|}{ Death-3 } \\
\hline & \multicolumn{3}{|c|}{ Discovery $(n=232)$} & \multicolumn{3}{|c|}{ Replication ( $n=99$ ) } \\
\hline Group & No, $n$ & Yes, $n$ & $\%$ Yes & No, $n$ & Yes, $n$ & $\%$ Yes \\
\hline 3 (high) & 40 & 17 & 29.82 & 15 & 11 & 42.31 \\
\hline 2 (intermediate) & 130 & 21 & 13.91 & 54 & 13 & 19.40 \\
\hline 1 (low) & 23 & 1 & 4.17 & 6 & 0 & 0.00 \\
\hline \multicolumn{7}{|l|}{ Death-12 } \\
\hline & \multicolumn{3}{|c|}{ Discovery $(n=204)$} & \multicolumn{3}{|c|}{ Replication ( $n=93$ ) } \\
\hline Group & No, $n$ & Yes, $n$ & $\%$ Yes & No, $n$ & Yes, $n$ & $\%$ Yes \\
\hline 3 (high) & 35 & 19 & 35.19 & 14 & 11 & 44.00 \\
\hline 2 (intermediate) & 103 & 23 & 18.25 & 47 & 15 & 24.19 \\
\hline 1 (low) & 22 & 2 & 8.33 & 6 & 0 & 0.00 \\
\hline
\end{tabular}

Discovery, trajectory groups depicted in Fig. 2a; Replication, trajectory groups depicted in Fig. 2c; GOS-3, Glasgow Outcome Scale at 3 months (favorable = 4-5, unfavorable=1-3); GOS-12, Glasgow Outcome Scale at 12 months (favorable = 4-5, unfavorable =1-3); Death-3, death at 3 months; Death-12, death at 12 months

associations between inferred trajectory groups at cg25713625 and acute outcomes of CV or DCI were observed.

In our replication analyses, a similar additive effect of inferred DNAm trajectory groups was observed as shown in Table 4. As in the discovery phase, the percentage of participants with unfavorable outcomes increased substantially with each inferred DNAm trajectory group. For example, in our replication sample, $0 \%$ of participants in the low DNAm trajectory group had unfavorable GOS at 3 months compared with $61.5 \%$ of participants in the high DNAm trajectory group.

In the formal replication association analyses presented in Table 5, based on the additive effect of trajectory groups shown in Table 4, the low and intermediate DNAm trajectory groups were combined to stabilize regression parameters (necessary due to the low group's small sample size). Our discovery phase findings replicated with an increased odds of unfavorable outcomes observed in the high DNAm group compared with the low/intermediate DNAm groups. Specifically, we
Table 5 Replication results of binary logistic regression examining associations of cg25713625 (STEAP3) with patient outcomes while controlling for age, sex, self-identified race, and Fisher grade

Replication, polynomial order 002, Fig. 2c

Group 3 (high) vs. combined group 1 (low) and group 2 (intermediate)

\begin{tabular}{llll}
\hline Outcome & $\mathbf{O R}$ & $\mathbf{9 5 \%} \mathrm{Cl}$ & $\boldsymbol{p}^{\mathbf{c}}$ \\
\hline GOS-3 & 8.190 & 2.51 to 31.85 & $\mathbf{0 . 0 0 1 0 ^ { \mathbf { a } }}$ \\
GOS-12 & 6.257 & 1.85 to 24.53 & $\mathbf{0 . 0 0 4 7 ^ { \mathbf { a } }}$ \\
Death-3 & 2.253 & 1.26 to 4.23 & $\mathbf{0 . 0 0 8 0 ^ { \mathbf { a } }}$ \\
Death-12 & 2.028 & 1.10 to 3.92 & ${\underline{0.0272^{\mathrm{b}}}}$
\end{tabular}

Corresponds to trajectory groups depicted in Fig. 2c; GOS-3, Glasgow Outcome Scale at 3 months (unfavorable =1-3); GOS-12, Glasgow Outcome Scale at 12 months (unfavorable $=1-3$ ); Death-3, death at 3 months; Death-12, death at 12 months. a Significant associations (meeting empirical significance level) have been bolded. ${ }^{b}$ Suggestive associations (unadjusted $p<0.05$ ) have been underlined. 'Empirical significance threshold $=0.0241$ (calculated based on the minimum of $4 p$-values in permutation testing) 
observed significant associations with unfavorable GOS at 3 and 12 months $(O R=8.19, p=0.001$ and $O R=$ $6.257, p=0.005$, respectively) and death at 3 months $(O R=2.25, p=0.008)$. A suggestive association was also observed with death at 12 months $(O R=2.03, p=0.027)$ though it did not reach our empirical significance threshold of 0.0241 to adjust for multiple testing.

In post hoc cross-sectional analyses, we observed a persistent association between continuous DNAm at the STEAP3 site (ignoring trajectory groups) and patient outcomes at time 2 (days 3 to 5 post-aSAH), time 3 (days 6 to 8 post-aSAH), and time 4 (days 9 to 11 postaSAH), with higher DNAm being associated with an increased odds of unfavorable long-term outcomes (Table S9). As described in the Materials and methods section, we also had genome-wide blood DNAm data available in a subset of 67 participants on days 1 to 2 post-aSAH. To understand the potential utility of blood as a surrogate for CSF, we explored the correlation between cg25713625 CSF and blood DNAm data in the subset of participants with both tissues (Table S10) and observed a small to moderate correlation $(R=0.36, p=0.0029$, Fig. S4). In formal logistic regression analyses, no significant associations between continuous DNAm in neither CSF nor blood were observed on days 1-2 post-aSAH in the small subset of participants with both tissues (Table S11).

\section{cg08866780 (APP)}

For $\operatorname{cg} 08866780(A P P)$, in the discovery phase base model, we inferred three flat trajectory groups including a low DNAm group (group 1, 24.2\%), intermediate DNAm group (group 2, 53.5\%), and high DNAm group (group 3, 22.3\%) (Fig. 2d). Even after correcting for $\mathrm{CTH}$, we observed very similar trajectory patterns at this CpG site (Fig. 2e). Participant characteristics by trajectory group are presented in the SI (Tables S7 and S8). In discovery phase association analyses, significant associations were identified with favorable GOS at 12 months $(p=0.0001)$ and survival at 3 and 12 months $(p=0.001$ and $p=0.0006$, respectively) and a suggestive association was identified with GOS at 3 months $(p=0.006)$. Overall, we observed that participants in the intermediate DNAm group (group 2) had a decreased odds of unfavorable outcomes compared with low and high DNAm groups (groups 1 and 2, respectively) (Table $2 b$ ). These results were consistent even after correction for $\mathrm{CTH}$ with suggestive associations identified between inferred trajectory groups and favorable GOS at 3 and 12 months ( $p=0.004$ and $p=0.01$, respectively) and survival at 3 and 12 months $(p=0.02$ and $p=0.01$, respectively) (Table 2). No significant associations between inferred trajectory groups at cg08866780 and acute outcomes of CV or DCI were observed.
In our replication analyses, the observed structure did not replicate in any of the three APP sites evaluated (Fig. $2 \mathrm{f}$ and Fig. S5) nor did the models meet our post-GBTA diagnostic criteria (Table S12). Therefore, no APP sites were carried forward for patient outcome association testing in the replication phase.

\section{cg08553327 (TNF)}

Finally, for cg08553327 (TNF), in the discovery base models (i.e., unadjusted for $\mathrm{CTH}$ ), we inferred three trajectory groups including a low DNAm group (group 1, $67.3 \%$ ), intermediate DNAm group (group 2, 26.9\%), and high DNAm group (group 3, 5.8\%). In contrast to the CpG sites discussed above, DNAm at this site was quite dynamic after aSAH (Fig, 2g) and the TNF gene housed a hotspot of several CpG sites that were significantly associated with patient outcomes after aSAH and had similar trajectory shapes as shown in the SI (Table S5 and Additional File 2). CpG site cg08553327 was specifically chosen for replication because it had the smallest $p$-value in the gene and was amenable to MethylSeq. While the CTH-adjusted trajectory model at cg08553327 did not meet our post-GBTA diagnostic criteria, two other sites within the hotspot did and resulted in three steadily decreasing trajectory groups. At these sites, after correction for $\mathrm{CTH}$, suggestive associations persisted between inferred trajectory groups and GOS at 3 months (Additional File 2). Participant characteristics by trajectory group are presented in the SI (Tables S7 and S8). In discovery phase association analyses, significant associations were identified with unfavorable GOS at 3 months $(p=0.0003)$ and suggestive associations were identified with unfavorable GOS at 12 months $(p=0.02)$ and death at 3 and 12 months ( $p=0.009$ and $p=0.004$, respectively). Overall, we observed that participants in both the intermediate and high DNAm groups had an increased odds of unfavorable outcomes (Table 2c). No significant associations between inferred trajectory groups at cg08553327 and acute outcomes of CV or DCI were observed.

In our replication analyses, the observed structure in the discovery data partially replicated in shape, but not group membership at TNF site 3 (Fig. 2h) but not in sites 1, 2, or 4 (Fig. S6). Only the trajectory models for sites 3 and 4 met our post-GBTA diagnostic criteria (Table S12) and were carried forward for patient outcome association testing. None of the observed associations with patient outcomes replicated (Table S13).

\section{Discussion}

Examining DNAm of CSF, a tissue that is proximal to the central nervous system and drained as part of clinical management to reduce intracranial pressure post-aSAH, offered a unique insight into patient recovery, 
particularly in the context of iron homeostasis posthemorrhage. Of the candidates examined, associations from three $\mathrm{CpG}$ sites in three genes were examined in replication testing including cg25713625 (STEAP3), $\operatorname{cg} 08866780(A P P)$, and $\operatorname{cg} 08553327$ (TNF). Of these, our replication analyses supported nearly identical inferred DNAm trajectory groups and relationships with patient outcomes for cg25713625 (STEAP3, UCSC Genome Browser GRCh37/hg 19, chr2: 120022835). Specifically, as the inferred DNAm trajectory group level increased, participants had an increased odds of unfavorable GOS and death at 3 and 12 months.

STEAP3 was selected as a candidate gene for this study given its primary role in iron transport and homeostasis. Specifically, metalloreductase STEAP3 (Steap3), also known as six-transmembrane epithelial antigen of prostate 3, the protein encoded by the STEAP3 gene, converts the stable but insoluble ferric form of iron to a less stable but soluble ferrous form [24]. Steap3 deficiency leads to impaired iron homeostasis and presents clinically as microcytic anemia with iron overload [24]. In addition to iron overload as a primary mechanism contributing to ferroptosis, accumulation of lethal, lipidbased reactive oxygen species has also been found to be important $[25,26]$. Specifically, elevated free iron postaSAH has great potential to cause oxidative degradation of lipids that make up cell membranes. For example, in animal models of subarachnoid hemorrhage, elevated iron caused an increase in lipid peroxides and administration of ferrostatin-1, a lipophilic antioxidant which protects cell membranes from lipid oxidation, in turn decreased free iron and improved lipid peroxidation [25]. This intervention successfully prevented both ferroptosis and early brain injury, but had no effect on apoptosis [25]. Beyond this preclinical work, the role of lipid peroxidation metabolites (e.g., 20-HETE) has been associated with unfavorable outcomes after aSAH in humans [27]. Increased levels of Steap3, though essential to normal iron homeostasis, can result in degradation of the cellular membrane through lipid peroxidation, leading to failure of hemolysis and clearance of red blood cells [28], further highlighting the potential importance of this gene.

In addition to iron homeostasis and lipid peroxidation, knockdown of STEAP3 has been shown to inhibit cell progression suggesting this protein may also play a role in downstream responses to $\mathrm{p} 53$, including promoting apoptosis [29]. Steap3 is also thought to be important in immunity as iron deficiency confers resistance to the risk of infection [24]. STEAP3, the only STEAP family member to be highly expressed in macrophages, has been shown to be down-regulated by lipopolysaccharide administered as a surrogate for infection in rats [24]. Our CpG site of interest, cg25713625, is annotated to a south shore in the 3' untranslated region of STEAP3. Aside from associations with age in whole blood in children [30-32], there is no significant literature directly related to cg25713625. Interestingly, however, STEAP3 has been identified as a differentially expressed gene in the intracranial aneurysm wall compared with a control tissue of the superficial temporal artery [33]. Specifically, in a study of 6 participants with unruptured intracranial aneurysms, the authors observed a fold change $>2.5$ with higher STEAP3 expression observed in aneurysm tissue [33].

A surprising finding of this study was that, with the exception of the low DNAm trajectory group in the replication sample, we observed little change over time in the trajectories inferred at this CpG site (Fig. 2a-c). These data suggest that DNAm at this site may not respond dynamically as hypothesized, at least acutely postaSAH, and that longitudinal data are likely not needed for use as a clinical biomarker. We confirmed this in post hoc cross-sectional analyses in which we observed largely persistent associations between continuous DNAm (ignoring trajectory groups) and long-term outcomes (Table S9). Though these associations were not significant at cross-sectional time 1 (days 1 to 2 postaSAH) or cross-sectional time 5 (days 12 to 13 postaSAH), the odds ratios were generally all in the expected direction. Notably, even when correcting for CTH in our discovery analyses, similar associations were observed at this CpG site, suggesting the association is not dependent on changing cell type proportions, further increasing its potential clinical utility.

In comparing cg25713625 DNAm in blood and CSF, we observed only a small to moderate correlation, suggesting blood might not be an appropriate surrogate for this STEAP3 CSF biomarker (Fig. S4). In our formal regression exploring this further, while there were no significant associations in either tissue, the odds ratios were in the expected direction in CSF $(O R>1)$, but not blood $(O R<1)$, further supporting the idea that blood might not be an appropriate surrogate (Table S11). In any case, these exploratory post hoc analyses were conducted in a small subset of the data ( $n=67$ participants), which greatly limits the interpretability. Studies in larger samples are needed.

We also selected $A P P$ as a candidate gene for inclusion in this study as amyloid precursor protein is highly expressed in the human brain and ubiquitously expressed across many human tissues [34, 35]. Specific to iron homeostasis, the role of APP in managing iron levels is feedback-regulated as iron influx is an important driver in translational expression of neuronal $A P P$ via an iron-responsive element and, in turn, $A P P$ plays a role in iron efflux by stabilizing the heavy subunit of ferritin and ferroportin-1 at the binding site [34]. Likewise, 
TNF was selected for inclusion as tumor necrosis factor is a pro-inflammatory cytokine most commonly recognized for its role in inflammation [36]. Iron homeostasis and inflammation are intimately tied as iron deficiency confers resistance to risk of infection and improves inflammatory conditions (e.g., anemia of inflammation in chronic disease). TNF plays an important role in iron homeostasis and host defense through regulating inflammation and hypoferremia [37]. Based on our discovery analyses, sites in APP (cg08866780, UCSC Genome Browser GRCh37/hg 19, chr21: 27543523) and TNF (cg08553327, UCSC Genome Browser GRCh37/hg 19, chr6: 31543647) were selected for replication. We were unable to replicate our findings, however, suggesting DNAm at the two sites examined are not likely important biomarkers or clinical targets post-aSAH.

\section{Strengths and limitations}

There were many strengths to this study. First, the DNAm data were quite unique and available in a relatively large sample of aSAH participants compared with other patient outcome studies post-aSAH. In addition to being longitudinal in nature spanning the acute recovery period post-aSAH, these data were generated from postaSAH CSF, an understudied tissue. Likewise, we had blood DNAm data in a subset of participants for comparison. Using these data and our objective GBTA approach, we were able to identify dynamic changes in CSF DNAm following aSAH. Furthermore, because genome-wide DNAm data existed in our discovery sample, we were able to examine the effects of $\mathrm{CTH}$. CTH is critical in biological interpretation of DNAm studies as differences in cell type proportions within biosamples can impact the overall DNAm level. In the case of post-aSAH CSF, biological interpretation of results presents even greater challenges given the immediate contamination of CSF by peripheral blood, vessel tissue, and brain tissue, but gradual clearing during the acute recovery period. In the case of clinical interpretation, however, it matters less if the biomarker association is attributed to DNAm related to changing $\mathrm{CTH}$ for that site - or simply the site itself without regard to $\mathrm{CTH}-$ as long as a signal can be replicated. While $\mathrm{CTH}$ can be controlled for if genome-wide DNAm data are collected [38], it is currently not practical to do so for a clinical biomarker given the associated expense of either cellsorting or generating genome-wide DNAm data.

Additional strengths of this study included rigorous data QC, imbedded replication, and collection of validation samples (i.e., DNAm data collected for 78 samples from 22 participants using both the discovery and replication platforms). In examining these data, we observed a strong correlation between discovery and replication DNAm data $(R=0.74)$, though the replication data were shifted up by approximately $10 \%$ (Fig. S7). Furthermore, in contrast to traditional GBTA, which requires iterative modeling and subjective decision-making from researchers, the GBTA applied here was objective and reproducible as it was largely automated and used a predefined decisionmaking tree and modeling protocol as detailed elsewhere [9]. Not only did we replicate both the structure and associations observed for the STEAP3 site in an independent sample of participants, but we were also able to perform a sensitivity GBTA which included the 22 overlapping validation participants. Of the 22 participants, 16 were assigned to the same group in the replication phase as in the discovery phase. While 8 participants shifted to a new group in the replication (Fig. S8), in all cases, it was an adjacent group (e.g., low to intermediate rather than low to high), further highlighting the replicability of our protocol.

Despite these strengths, there are some important limitations. First, this study was primarily made up of participants who self-reported their race as White. Although the distribution of race observed is largely consistent with Southwestern Pennsylvania demographics, this limits the generalizability of our findings in other races, ethnicities, and ancestries. These factors should be examined in larger and more diverse samples in the future. Next, we used a candidate gene approach which has important weaknesses [39]. However, we addressed the major concerns of candidate gene studies by carefully correcting for multiple testing and replicating our findings in an independent test sample of participants.

While the objective nature of our automated protocol was an important strength of this study, it also eliminated a large portion of CpG sites at which we could not reliably infer trajectory groups. As such, there may be important signals in these data that were not examined in association testing with patient outcomes. Similarly, our tier 1 analyses were limited to existing genome-wide DNAm data. As part of our genome-wide DNAm data QC pipeline described below, CpG sites located on the $\mathrm{X}$ chromosome were removed. Therefore, data for two of our candidate genes (HEPH and HJV) were not available for analysis despite their roles in iron homeostasis. It is possible that variability in these genes is important to outcomes after aSAH. Finally, due to budgetary limitations, we could not collect replication data for all $\mathrm{CpG}$ sites that met significance after correction for multiple testing. While we attempted to be as objective as possible in our prioritization of results for replication, true and 
important signals may exist for other CpG sites. These sites should be examined in the future, particularly those highlighted in Table S5.

\section{Conclusion}

The results of this study support a role for DNAm of cg25713625 (STEAP3) in recovery following aSAH. Based on the inferred DNAm trajectories at cg25713625 observed here, we conclude that longitudinal data are not required as CSF DNAm of this site did not appear to change significantly post-aSAH. Additional research, including functional studies, is needed to better understand ferroptosis post-aSAH and further explore the potential role of CSF DNAm of cg25713625/STEAP3 as a biomarker of unfavorable outcomes, or therapeutic target to improve outcomes, to translate these findings clinically.

\section{Materials and methods}

\section{Study design, setting, and sample}

This was an ancillary, observational, candidate gene association study that capitalized on an existing cohort of aSAH participants with longitudinal phenotype data, biosamples, and genome-wide DNAm data for a subset of participants (Fig. 1) [40]. This study assessed the relationship between inferred DNAm trajectories of iron homeostasis candidate genes and patient outcomes acutely (first 2 weeks post-aSAH) and in the long-term (at 3 and 12 months post-aSAH) using a two-phase design (targeted discovery and replication).

This study adhered to all ethical considerations and was approved by the Institutional Review Board of the University of Pittsburgh. Following informed consent, participants were prospectively recruited from UPMC Presbyterian neurovascular intensive care unit in Pittsburgh, PA, between 2000 and 2017 if they (1) were aged 18 years and older, (2) were newly diagnosed ( $\leq 5$ days) with aSAH verified with cerebral angiogram, (3) had a Hunt and Hess grade $\geq 2$ and/or Fisher grade $\geq 1$, (4) were able to read/speak English, and (5) had no history of debilitating neurological disorders. Additional inclusion criteria for this ancillary study included ventriculostomy insertion as part of clinical management to supply CSF samples and availability of serial CSF samples across 14 days post-aSAH.

\section{DNA methylation data \\ Discovery phase}

Data collection The discovery phase capitalized on existing, longitudinal, genome-wide DNAm data collected for 273 participants at up to five time points over 14 days following aSAH as previously described [41] (Fig. 1). DNAm data were generated from DNA extracted from bagged CSF collected as a standard treatment and changed daily for 14 days following aSAH by trained study staff using sterile procedures. The CSF samples were centrifuged, and the cellular pellet and supernatant were stored at $-80^{\circ}$ until DNA extraction. DNA was extracted from the cellular pellet using the QIAmp Midi kit (catalog \#51185) from Qiagen Corp (Qiagen, Valencia, CA, USA), DNA concentration and quality checks were completed, and bisulfite conversion was performed. Genome-wide DNAm data were generated and scanned using the Infinium HumanMethylation450 Beadchip and Illumina iSCAN (Illumina, Incorporated, San Diego, CA, USA) at Johns Hopkins University's Center for Inherited Disease Research.

As part of our laboratory QC procedures, samples were placed across 11 plates using several strategies to reduce the impact of technical artifacts as described in detail elsewhere [41]. Technical replicates and DNAm control samples were included to assess the reliability of data. Raw genome-wide DNAm data were analyzed using Genome Studio Software (Illumina, Incorporated, San Diego, CA, USA). In parallel, for a subset of 88 participants, genome-wide DNAm data were generated from peripheral blood at a single time point, targeting the early admission period, as detailed elsewhere [41]. In post hoc analyses detailed below, these data were used to compare DNAm in CSF and blood for our top hits, limiting the data to overlapping CSF and blood samples available on days 1-2 post-aSAH for 67 participants.

Genome-wide data QC Genome-wide data cleaning and QC was implemented using $\mathrm{R}$ packages minfi [42] and ENmix [43]. This pipeline included removal of poorly performing and outlying samples based on bisulfite control intensities and detection $p$-values and background and dye bias correction to remove non-specific signals from the DNAm data $[43,44]$. To further reduce technical variation related to Infinium $450 \mathrm{~K}$ platform chemistry (i.e., differences in type I and type II probes) and batch effects (i.e., chip, row, and column effects), functional normalization was performed using the "preprocessfunnorm" function from the minfi package [42, 45]. Additional details of our QC pipeline are summarized elsewhere [41]. Of the available sample, observations from days 0 and 14 were removed due to low sample counts, and 13 participants were dropped because they had only one DNAm data observation which precluded trajectory analysis. Our final discovery sample size was 260 participants.

Cell type heterogeneity Under normal conditions, CSF is clear and contains few cells. Following aneurysm rupture, however, CSF is contaminated with both peripheral blood and tissue from the ruptured aneurysm, which 
gradually clears as the CSF renews during recovery. Because no reference-based methods exist to control for $\mathrm{CTH}$ in post-aSAH CSF, we explored this in detail in the discovery phase by generating estimated cell type proportions from the genome-wide DNAm data using Houseman's reference-free method [46]. This results in estimated proportions of five putative "cell types" for each biosample which were used in our analyses as detailed below.

DNAm data extraction Thirty-nine candidate genes were selected based on their known biological roles in iron homeostasis and described in detail as part of our previous, related work $[9,10]$. DNAm data for our candidate genes were extracted from the gene transcript region \pm 2000 base pairs upstream and downstream. Data for two genes (HEPH and PGRMC1) were not available in the cleaned genome-wide data and data for the hepcidin gene (HAMP) were analyzed in the pilot work to develop the protocols for this study (Table S1) [9].

\section{Replication phase}

Prioritization of top hits for replication Following gene-specific data screening and analysis described below, top hits for replication were prioritized based on the strength of the identified associations, consistency of results after adjustment for $\mathrm{CTH}$, and presence of hotspots (i.e., multiple $\mathrm{CpG}$ sites near each other associated with outcomes) as detailed in the SI (Fig. S1).

DNAm data collection and QC The replication sample was recruited together with the discovery sample as part of a larger parent cohort (Fig. 1). Longitudinal replication DNAm data were generated from CSF for our top hits using pyrosequencing (i.e., MethylSeq) at the Center for Inherited Disease Research. Using $500 \mathrm{ng}$ of DNA, bisulfite conversion was carried out with the Qiagen EpiTect Bisulfite kit and PCR reactions were completed using the Qiagen Pyromark PCR Kit following standard protocols and assays designed by and proprietary to Qiagen. Data were generated using Qiagen Pyromark Q48 Autoprep instrument and were called using the Pyromark Q48 Autoprep 2.4.2 software. Probe sequences were designed to capture top hits and regions surrounding top hits. As part of our QC filtering, sites with > 5\% of samples failing MethylSeq were excluded. Individual samples with incomplete conversion were not used for sequencing and individual samples that failed the MethylSeq assay were excluded. Additional replication data collection details are provided in the SI (Section II). In total, MethylSeq data were generated for 122 participants, 22 of which overlapped with the discovery sample for validation purposes. The final replication sample size (i.e., independent participants) was 100 participants (Fig. $1)$.

\section{Patient outcomes}

Acute patient outcome measures included the occurrence or absence of CV and DCI within a 14-day window following aSAH. CV was defined as $\geq 25 \%$ cerebral vessel narrowing measured by a neurosurgeon via cerebral angiogram obtained as clinically necessary [9], and DCI was defined as the co-occurrence of neurological deterioration (e.g., new and persistent $[>1 \mathrm{~h}]$ neurological deficit) and abnormal cerebral blood flow measured using cerebral angiogram or transcranial Doppler (performed twice a day) [9]. Acute outcomes of CV and DCI were treated as binary (occurrence/absence).

Long-term patient outcome measures included the GOS and death at 3 and 12 months following aSAH. These data were collected by trained study staff via inperson or telephone interviews. The GOS is an indication of a participant's ability to function on a scale of 1 (death) to 5 (good recovery) and has established validity in people with neurological injury [47]. GOS was dichotomized as good (scores of 4 to 5 ) or unfavorable (scores of 1 to 3). Death data were obtained from the medical record, caregiver/family report, or the Social Security Death Index and treated as binary (yes/no) at the outcome timepoint of interest. In cases where participants were unable to take part in the interview, a caregiver or family member knowledgeable about the participant's ability to function was interviewed. All study staff involved in outcome data collection were blinded to DNAm status.

Finally, this study capitalized on existing confounding/ covariate data extracted from the medical record or collected in surveys as part of a larger research study. These demographic and clinical data included age, sex, race, treatment/intervention, and Fisher grade which provides a measure of the initial extent of aSAH injury based on the amount and distribution of blood observed on a computed tomography scan.

\section{Statistical analyses \\ Preliminary analyses}

All statistical analyses were conducted using $\mathrm{R}$ [48] and SAS (SAS Institute Incorporated, Cary, NC, USA). Standard descriptive statistics were computed for all independent, dependent, and potentially confounding/covariate data given the variable's level of measurement. Preliminary analyses were conducted to identify potential confounders/covariates. DNAm data were analyzed as $M$ values and any value labeled as an extreme outlier (a DNAm value above or below three times the interquartile range) was replaced with the maximum or minimum observed DNAm value below the extreme outlier 
threshold for values on each day. Missing data were assessed and filled in from the medical record when possible. An expanded description of our statistical analyses is provided in the SI (Section III).

\section{Group-based trajectory analyses}

GBTA, an unsupervised clustering method used to identify clusters of individuals who have similar development trajectories (e.g., DNA methylation trajectory groups post-aSAH), was conducted using Proc TRAJ and a censored normal model in SAS. While other methods of longitudinal modeling (e.g., latent curve analysis) estimate the sample average trajectory and use covariates to explain variability about this average, GBTA is dissimilar in that it assumes the sample is composed of distinct groups, each with a different underlying trajectory. GBTA is a highly flexible approach, allowing for a variety of complexities including missing or sparse data, time-varying covariates, and small sample sizes as it uses the maximum likelihood method to estimate parameters, including group sizes and shapes of trajectories $[49,50]$. Therefore, GBTA is an ideal approach to apply in cases where individual observations are available from different time points (e.g., different days post-aSAH) as it generates asymptotically unbiased parameter estimates.

In GBTA, models with a varying number of groups and polynomial orders (i.e., group trajectory shapes) are compared to find the model that best fits the longitudinal data [51]. As part of this process, a trajectory group-specific posterior probability of assignment is computed for each participant. Ultimately, individuals are assigned to the group for which their posterior probability is the highest [51]. Given the subjectivity and iterative modeling required in traditional GBTA, and the large number of candidate genes and $\mathrm{CpG}$ sites examined here, the model selection process was largely automated. Specifically, we determined the "best fitting" of 39 possible models with a maximum of three groups and comprehensive combinations of polynomial orders of 0 (intercept only), 1 (linear), and 2 (quadratic). Our GBTA automated protocol has been described in detail as part of our pilot work [9] and summarized in the SI (Section II).

Following GBTA for each CpG site, we performed a secondary evaluation of model adequacy (i.e., evaluation of post-GBTA diagnostics) using several traditional measures including (1) an average posterior probability (AvePP) > 0.7, (2) odds of correct classification (OCC) > 5 , (3) estimated group membership $(\pi)>5 \%$, (4) reasonably close estimated group membership $(\pi)$ versus the assigned group proportion $\left(\mathrm{P}^{*}\right)$, and (5) a relatively narrow $95 \%$ confidence interval for the estimated group probability $(\pi)[9,49]$. If the trajectory model for an individual CpG site failed for the top two "best" models, we concluded that DNAm trajectory groups could not be inferred with high accuracy for that site and it was excluded from further analysis [9].

To examine the potential clinical utility of DNAm trajectories unadjusted for $\mathrm{CTH}$, as well as to support the biological interpretation of results evaluating potential confounding by cell type, we implemented our GBTA protocol twice for all $\mathrm{CpG}$ sites to compute DNAm trajectories both unadjusted for CTH (i.e., base models) and adjusted for CTH [9]. CTH data were generated in our QC pipeline as described above and were controlled for as time-varying covariates during GBTA in our $\mathrm{CTH}$-adjusted models, embedding the additional variables within the trajectory group assignment. GBTA was performed using $M$ values and final models and estimates were converted to beta values for presentations in trajectory plots.

Patient outcome association testing Binary logistic regression was performed in $\mathrm{R}$ to determine the relationship between inferred trajectory groups for each $\mathrm{CpG}$ site and patient outcomes while controlling for age, sex, race, and Fisher grade (i.e., Outcome $\sim$ Trajectory group + age + sex + race + Fisher grade). A partial $F$ test was used to produce a global $p$-value of the overall model fit by comparing the full model (including the trajectory group) with a restricted model (omitting the trajectory group). As detailed in the SI (Section III), permutation testing was used to correct for multiple testing of correlated outcomes [9]. Associations with a $p$-value $<0.05$ were considered suggestive, and associations with a $p$ value falling below the empirical significance threshold were considered significant.

\section{Post hoc analyses}

Longitudinal DNAm data were collapsed into crosssectional time points including time 1 (days 1-2 postaSAH), time 2 (days 3 to 5 post-aSAH), time 3 (days 6 to 8 post-aSAH), time 4 (days 9 to 11 post-aSAH), and time 5 (days 12 to 14 post-aSAH). Binary logistic regression was performed to determine the relationship between continuous DNAm of top hits (i.e., ignoring trajectory groups) and patient outcomes while controlling for age, sex, race, and Fisher grade within crosssectional time points. For the subset of participants with both CSF and blood DNAm data on days 0-2 postaSAH ( $n=67$ participants), $M$ values and beta values were compared using scatterplots and Pearson correlation coefficients, and regression lines were drawn to better understand this relationship relative to $y=x$. Finally, binary logistic regression was again performed to explore the relationship between continuous DNAm of top hits in CSF and blood and patient outcomes while controlling for age, sex, race, and Fisher grade. 


\section{Abbreviations}

aSAH: Aneurysmal subarachnoid hemorrhage; DNAm: DNA methylation; CSF: Cerebrospinal fluid; CTH: Cell type heterogeneity; CV: Cerebral vasospasm; DCl: Delayed cerebral ischemia; GBTA: Group-based trajectory analysis; SI: Supplementary information; STEAP3: STEAP3 metalloreductase; APP: Amyloid precursor protein; TNF: Tumor necrosis factor; OR: Odds ratio; QC: Quality control; OCC: Odds of correct classification; AvePP: Average posterior probability

\section{Supplementary Information}

The online version contains supplementary material available at https://doi. org/10.1186/s43682-021-00003-5.

\section{Additional file 1: Section I. Expanded results; Table S1. Iron}

homeostasis gene, data extraction, and extreme outlier summary; Table S2. Number of DNA methylation observations by day in discovery and replication samples; Table S3. Summary of significant and suggestive associations for base models (no adjustment for (TH); Table S4. Summary of significant and suggestive associations for CTH-adjusted models; Figure S1. Flow chart for prioritization of findings for replication; Table S5. Summary of prioritization of top hits in base models (i.e., unadjusted for $\mathrm{CTH}$ to allow for replication by MethySeq); Figure S2. Discovery phase spaghetti plots depicting DNA methylation over 13 days post-aSAH for top hits in STEAP3, APP, and TNF; Table S6. Discovery and replication levels of DNA methylation as measured by beta values (corresponds with Figure 2); Table S7. Discovery phase participant characteristics by trajectory group for cg25713625 (STEAP3), cg08866780 (APP), and cg08553327 (TNF); Table S8. Discovery phase patient outcome distributions by base model trajectory groups for cg25713625 (STEAP3), cg08866780 (APP), and cg08553327 (TNF); Figure S3. Sankey plots depicting shifts in trajectory group assignment between discovery phase base and CTH-adjusted models; Table S9. Results of discovery phase binary logistic regression examining associations of continuous cg25713625 (STEAP3) CSF DNA methylation with patient outcomes while controlling for age, sex, self-identified race, and Fisher grade in cross-sectional time points; Table S10. Characteristics of the subset of participants with blood DNA methylation available on days 0-2 post-aSAH; Figure S4. Correlation between cg25713625 (STEAP3) CSF and Blood DNA Methylation, Days 0-2 post-aSAH; Table S11. Results of binary logistic regression examining associations of continuous cg25713625 (STEAP3) DNA methylation with patient outcomes while controlling for age, sex, self-identified race, and Fisher grade in the subset of participants with both blood and CSF available on days 0-2 post-aSAH in the discovery sample; Figure S5. Replication DNA methylation trajectory plots for CpGs near cg08866780 (APP); Figure S6. Replication DNA methylation trajectory plots for CpGs near cg08553327 (TNF); Table S12. Post-GBTA diagnostic summary for replication data group-based trajectory analysis for sites in STEAP3, APP, and TNF; Table S13. Replication results of binary logistic regression examining associations of cg08553327 (TNF), sites 3 and 4, with patient outcomes while controlling for age, sex, self-identified race, and Fisher grade; Figure S7. Comparison of cg25713625 (STEAP3) DNA methylation data from validation samples overlapping between discovery and replication data; Figure S8. Sankey plot depicting shifts in trajectory group assignment between discovery and replication analyses for cg25713625 (STEAP3) replication; Section II. Expanded replication data collection methods; Table S14. Replication assay information for top hits; Table S15. Summary of replication data QC (pass, check, fail); Section III. Expanded statistical analysis.

Additional file 2:. Gene-specific detailed results.

\section{Acknowledgements}

We would like to thank the participants and their families for their involvement in this research; Dr. John Shaffer for his contributions to the parent DNA methylation project; Dr. Dongjing Liu and Annie Arockiaraj for their contributions to the DNA methylation data quality control pipeline; and the anonymous reviewers who took the time to critically evaluate this paper as their feedback improved the quality and clarity of our work.

\section{Authors' contributions}

LWH and YPC conceived and designed the present study with additional methodological feedback from all authors. YPC is the principal investigator of the parent study for this project and was responsible for the acquisition of the discovery DNA methylation data (including funding). LWH is the principal investigator of the present study and, along with YPC, was responsible for the acquisition of the replication DNA methylation data (including funding). PRA, EAC, SMP, and SAA were responsible for the development of the parent cohort from which the biosamples and phenotype data were extracted. SD was responsible for biospecimen handling and DNA extraction. DEW was responsible for the discovery genome-wide DNA methylation data quality control. LWH and DEW developed the GBTA protocol and LWH performed the statistical analyses. All authors contributed to the interpretation of the data and results. LWH drafted the initial manuscript and all authors reviewed, critically revised, and approved the final manuscript. All authors agree to be accountable for all aspects of the work.

\section{Funding}

Research reported in this publication was supported by the National Institute of Nursing Research and National Center for Advancing Translational Sciences of the National Institutes of Health under Award Numbers F31NR017311, R01NR004339, R01NR013610, T32NR009759, and TL1TR001858 with additional support from the Nightingale Awards of Pennsylvania, Center for Jonas Nursing and Veterans Healthcare, International Society of Nurses in Genetics, American Nurses Foundation (Eastern Nursing Research Society and Eleanor C. Lambertson Scholar Fund), and University of Pittsburgh Leslie A. Hoffman Endowed Research Award and Institute for Clinical Research and Education Career Development Award. The content is solely the responsibility of the authors and does not represent the official views of the National Institutes of Health or supporting foundations.

\section{Availability of data and materials}

The discovery data analyzed as part of this study are available in dbGAP, accession number: phs001990.v1.p1.

\section{Declarations}

Ethics approval and consent to participate

This study has an Institutional Review Board Approval from the University of Pittsburgh (study number STUDY19100368). Written informed consent was obtained from all participants at enrollment.

\section{Consent for publication}

Not applicable.

\section{Competing interests}

The authors declare that they have no competing interests.

\section{Author details}

${ }^{1}$ Department of Human Genetics, Graduate School of Public Health, University of Pittsburgh, Pittsburgh, PA 15261, USA. ²Department of Biostatistics, Graduate School of Public Health, University of Pittsburgh, Pittsburgh, PA, USA. ${ }^{3}$ Department of Acute and Tertiary Care, School of Nursing, University of Pittsburgh, Pittsburgh, PA, USA. ${ }^{4}$ College of Pharmacy, University of Texas at Austin, Austin, TX, USA. ${ }^{5}$ Department of Health Promotion and Development, School of Nursing, University of Pittsburgh, Pittsburgh, PA, USA.

Received: 15 July 2021 Accepted: 25 August 2021

Published online: 20 December 2021

\section{References}

1. Teo $\mathbf{M}$, Teo $\mathbf{M}$, Turner $\mathbf{C}$, et al. What factors determine treatment outcome in aneurysmal subarachnoid hemorrhage in the modern era? A post hoc STASH analysis. World Neurosurg. 2017;105:270-81. https://doi.org/10.1016/j. wneu.2017.05.005.

2. $\quad$ van Gijn J, Kerr RS, Rinkel GJE. Subarachnoid haemorrhage. Lancet. 2007; 369(9558):306-18. https://doi.org/10.1016/S0140-6736(07)60153-6.

3. Roos YBWEM, Dijkgraaf MGW, Albrecht KW, Beenen LFM, Groen RJM, de Haan RJ, et al. Direct costs of modern treatment of aneurysmal 
subarachnoid hemorrhage in the first year after diagnosis. Stroke. 2002;33(6): 1595-9. https://doi.org/10.1161/01.STR.0000016401.49688.2F.

4. Turi E, Conley Y, Stanfill AG. A literature review of psychosocial comorbidities related to working capacity after aneurysmal subarachnoid hemorrhage. J Neurosci Nurs. 2017;49(3):179-84. https://doi.org/10.1097/JNN. 0000000000000281.

5. Oka F, Chung DY, Suzuki M, Ayata C. Delayed cerebral ischemia after subarachnoid hemorrhage: experimental-clinical disconnect and the unmet need. Neurocrit Care. January 2019;32(1):238-51. https://doi.org/10.1007/s12 028-018-0650-5.

6. Gomes JA, Selim M, Cotleur A, Hussain MS, Toth G, Koffman L, et al. Brain iron metabolism and brain injury following subarachnoid hemorrhage: iCeFISH-pilot (CSF iron in SAH). Neurocrit Care. 2014;21(2):285-93. https://doi. org/10.1007/s12028-014-9977-8.

7. Ono S, Zhang ZD, Marton LS, Yamini B, Windmeyer E, Johns L, et al. Heme oxygenase-1 and ferritin are increased in cerebral arteries after subarachnoid hemorrhage in monkeys. J Cereb Blood Flow Metab. 2000; 20(7):1066-76. https://doi.org/10.1097/00004647-200007000-00006.

8. Suzuki H, Muramatsu M, Tanaka K, Fujiwara H, Kojima T, Taki W. Cerebrospinal fluid ferritin in chronic hydrocephalus after aneurysmal subarachnoid hemorrhage. J Neurol. 2006;253(9):1170-6. https://doi.org/10.1 007/s00415-006-0184-1.

9. Heinsberg LW, Arockiaraj Al, Crago EA, Ren D, Shaffer JR, Sherwood PR, Sereika SM, Weeks DE, Conley YP Genetic variability and trajectories of DNA methylation may support a role for HAMP in patient outcomes after aneurysmal subarachnoid hemorrhage. Neurocrit Care. 2020;32(2):550-563. doi:https://doi.org/10.1007/s12028-019-00787-4, 2

10. Heinsberg LW, Alexander SA, Crago EA, Minster RL, Poloyac SM, Weeks DE, et al. Genetic variability in the iron homeostasis oathway and patient outcomes after aneurysmal subarachnoid hemorrhage. Neurocrit Care. April 2020;33(3):749-58. https://doi.org/10.1007/s12028-020-00961-z.

11. Chen J, Wang Y, Wu J, Yang J, Li M, Chen Q. The potential value of targeting ferroptosis in early brain injury after acute CNS disease. Front Mol Neurosci. 2020;13:110. https://doi.org/10.3389/fnmol.2020.00110.

12. Ganz T. Hepcidin and iron regulation, 10 years later. Blood. 2011;117(17): 4425-33. https://doi.org/10.1182/blood-2011-01-258467.

13. Bishop GM, Robinson SR. Quantitative analysis of cell death and ferritin expression in response to cortical iron: implications for hypoxia-ischemia and stroke. Brain Res. 2001;907(1-2):175-87. https://doi.org/10.1016/S00068993(01)02303-4

14. Lee J-Y, Keep RF, Hua Y, Pandey A, Xi G. The role of iron in the brain following subarachnoid hemorrhage. In: Li YW, Zhang JH, eds. Metal lon in Stroke. ; 2012:273-282.

15. Dixon SJ, Lemberg KM, Lamprecht MR, Skouta R, Zaitsev EM, Gleason CE, et al. Ferroptosis: an iron-dependent form of nonapoptotic cell death. Cell. 2012;149(5):1060-72. https://doi.org/10.1016/j.cell.2012.03.042.

16. Lee J-Y, Keep RF, Hua Y, Ernestus R-I, Xi G. Deferoxamine reduces early brain injury following subarachnoid hemorrhage. Acta Neurochir Suppl. 2011;112: 101-6. https://doi.org/10.1007/978-3-7091-0661-7_18.

17. Yeatts SD, Palesch YY, Moy CS, Selim M. High dose deferoxamine in intracerebral hemorrhage (Hi-Def) trial: rationale, design, and methods Neurocrit Care. 2013;19(2):257-66. https://doi.org/10.1007/s12028-013-9861-y.

18. Meng H, Li F, Hu R, Yuan Y, Gong G, Hu S, et al. Deferoxamine alleviates chronic hydrocephalus after intraventricular hemorrhage through iron chelation and Wnt1/Wnt3a inhibition. Brain Res. 2015;1602(C):44-52. https:// doi.org/10.1016/j.brainres.2014.08.039.

19. Suzuki H, Muramatsu M, Kojima T, Taki W. Intracranial heme metabolism and cerebral vasospasm after aneurysmal subarachnoid hemorrhage. Stroke. 2003;34(12):2796-800. https://doi.org/10.1161/01.STR.0000103743.62248.12.

20. Felling RJ, Song H. Epigenetic mechanisms of neuroplasticity and the implications for stroke recovery. Exp Neurol. 2015;268:37-45. https://doi. org/10.1016/j.expneurol.2014.09.017

21. Baccarelli A, Wright R, Bollati V, Litonjua A, Zanobetti A, Tarantini L, et al. Ischemic heart disease and stroke in relation to blood DNA methylation. Epidemiology. 2010;21(6):819-28. https://doi.org/10.1097/EDE.0b013e3181f2 0457.

22. Zhang ZY, Zhang ZY, Fauser U, Schluesener HJ. Global hypomethylation defines a sub-population of reactive microglia/macrophages in experimental traumatic brain injury. Neurosci Lett. 2007;429(1):1-6. https:// doi.org/10.1016/j.neulet.2007.09.061
23. Hou Y, Zhang S, Wang L, Li J, Qu G, He J, et al. Estrogen regulates iron homeostasis through governing hepatic hepcidin expression via an estrogen response element. Gene. 2012;511(2):398-403. https://doi.org/10.1 016/j.gene.2012.09.060.

24. Zhang F, Tao Y, Zhang Z, Guo X, An P, Shen Y, et al. Metalloreductase Steap3 coordinates the regulation of iron homeostasis and inflammatory responses. Haematol Hematol J. 2012;97(12):1826. http://connection. ebscohost.com/c/articles/84662557/metalloreductase-steap3-coordinatesregulation-iron-homeostasis-inflammatory-responses-35. https://doi.org/1 0.3324/haematol.2012.063974.

25. Li Y, Liu Y, Wu P, Tian Y, Liu B, Wang J, et al. Inhibition of ferroptosis alleviates early brain injury after subarachnoid hemorrhage in vitro and in vivo via reduction of lipid peroxidation. Cell Mol Neurobiol. April 2020; 41(2):263-78. https://doi.org/10.1007/s10571-020-00850-1.

26. Fang Y, Gao S, Wang X, Cao Y, Lu J, Chen S, et al. Programmed cell deaths and potential crosstalk with blood-brain barrier dysfunction after hemorrhagic stroke. Front Cell Neurosci. 2020;14. https://doi.org/10.3389/ fncel.2020.00068.

27. Donnelly MK, Crago E. a, Conley YP, et al. 20-HETE is associated with unfavorable outcomes in subarachnoid hemorrhage patients. J Cereb Blood Flow Metab. 2015;35(9):1515-22. https://doi.org/10.1038/jcbfm.2015.75.

28. Howie HL, Hay AM, De Wolski K, et al. Differences in Steap3 expression are a mechanism of genetic variation of RBC storage and oxidative damage in mice. Blood Adv. 2019;3(15):2272-85. https://doi.org/10.1182/bloodadva nces.2019000605.

29. Han M, Xu R, Wang S, Yang N, Ni S, Zhang Q, et al. Six-transmembrane epithelial antigen of prostate 3 predicts poor prognosis and promotes glioblastoma growth and invasion. Neoplasia (United States). 2018;20(6):54354. https://doi.org/10.1016/j.neo.2018.04.002.

30. Xu C-J, Bonder MJ, Söderhäll C, Bustamante M, Baïz N, Gehring U, et al. The emerging landscape of dynamic DNA methylation in early childhood. BMC Genomics. 2017;18(1):25. https://doi.org/10.1186/s12864-016-3452-1.

31. Mulder RH, Neumann A, Cecil CAM, Walton E, Houtepen LC, Simpkin AJ, et al. Epigenome-wide change and variation in DNA methylation in childhood: trajectories from birth to late adolescence. Hum Mol Genet. 2021; 30(1):119-34. https://doi.org/10.1093/hmg/ddaa280.

32. Battram T, Yousefi P, Crawford G, et al. The EWAS Catalog: a database of epigenome-wide association studies. OSF Prepr. 2021. https://doi.org/1 0.31219/osf.io/837wn

33. Shi C, Awad IA, Jafari N, Lin S, du P, Hage ZA, et al. Genomics of human intracranial aneurysm wall. Stroke. 2009;40(4):1252-61. https://doi.org/10.11 61/STROKEAHA.108.532036.

34. Rogers JT, Venkataramani V, Washburn C, Liu Y, Tummala V, Jiang H, et al. A role for amyloid precursor protein translation to restore iron homeostasis and ameliorate lead (Pb) neurotoxicity. J Neurochem. 2016;138(3):479-94. https://doi.org/10.1111/jnc.13671

35. Nalivaeva NN, Turner AJ. The amyloid precursor protein: a biochemical enigma in brain development, function and disease. FEBS Lett. 2013;587(13): 2046-54. https://doi.org/10.1016/j.febslet.2013.05.010.

36. Nanami M, Ookawara T, Otaki Y, et al. Tumor necrosis factor-a-induced iron sequestration and oxidative stress in human endothelial cells. Arterioscler Thromb Vasc Biol. 2005;25(12).

37. Scaccabarozzi A, Arosio P, Weiss $G$, Valenti L, Dongiovanni P, Fracanzani AL, et al. Relationship between TNF-alpha and iron metabolism in differentiating human monocytic THP-1 cells. Br J Haematol. 2000;110(4): 978-84. http://www.ncbi.nlm.nih.gov/pubmed/1 1054092. . https://doi.org/1 0.1046/j.1365-2141.2000.02280.x.

38. Titus AJ, Gallimore RM, Salas LA, Christensen BC. Cell-type deconvolution from DNA methylation: a review of recent applications. Hum Mol Genet. 2017;26(R2):R216-24. https://doi.org/10.1093/hmg/ddx275.

39. Claussnitzer M, Cho JH, Collins R, Cox NJ, Dermitzakis ET, Hurles ME, et al. A brief history of human disease genetics. Nature. 2020;577(7789):179-89. https://doi.org/10.1038/s41586-019-1879-7.

40. Heinsberg L. Multi-omics of the iron homeostasis pathway in patient outcomes after aneurysmal subarachnoid hemorrhage [PhD Thesis]. 2020. http://d-scholarship.pitt.edu/39224/.

41. Arockiaraj Al, Liu D, Shaffer JR, Koleck TA, Crago EA, Weeks DE, et al. Methylation data processing protocol and comparison of blood and cerebral spinal fluid following aneurysmal subarachnoid hemorrhage. Front Genet. June 2020;11. https://doi.org/10.3389/fgene.2020.00671. 
42. Aryee MJ, Jaffe AE, Corrada-Bravo H, Ladd-Acosta C, Feinberg AP, Hansen $K D$, et al. Minfi: a flexible and comprehensive Bioconductor package for the analysis of Infinium DNA methylation microarrays. Bioinformatics. 2014; 30(10):1363-9. https://doi.org/10.1093/bioinformatics/btu049.

43. Xu Z, Niu L, Li L, Taylor JA. ENmix: a novel background correction method for Illumina HumanMethylation450 BeadChip. Nucleic Acids Res. 2016;44(3): e20. https://doi.org/10.1093/nar/gkv907.

44. Xu Z, Langie SAS, De Boever P, Taylor JA, Niu L. RELIC: a novel dye-bias correction method for Illumina Methylation BeadChip. BMC Genomics. 2017; 18(1):4. https://doi.org/10.1186/s12864-016-3426-3.

45. Fortin JP, Labbe A, Lemire M, Zanke BW, Hudson TJ, Fertig EJ, et al. Functional normalization of $450 \mathrm{k}$ methylation array data improves replication in large cancer studies. Genome Biol. 2014;15(11):503. https://doi. org/10.1186/s13059-014-0503-2.

46. Houseman EA, Molitor J, Marsit CJ. Reference-free cell mixture adjustments in analysis of DNA methylation data. Bioinformatics. 2014;30(10):1431-9. https://doi.org/10.1093/bioinformatics/btu029.

47. Jennett B, Bond M. Assessment of outcome after severe brain damage. Lancet (London, England). 1975;1 (7905):480-4 http://www.ncbi.nlm.nih.gov/ pubmed/46957.

48. Team RC. R: a language and environment for statistical computing. 2018. https://www.r-project.org/.

49. Nagin DS. Group-based modeling of development: Harvard University Press; 2005. https://doi.org/10.4159/9780674041318.

50. Jones BL, Nagin DS. Advances in group-based trajectory modeling and an SAS procedure for estimating them. Sociol Methods Res. 2007;35(4):542-71. https://doi.org/10.1177/0049124106292364.

51. Jones BL, Nagin DS, Roeder K. A SAS procedure based on mixture models for estimating developmental trajectories. Sociol Methods Res. 2001;29(3): 374-93. https://doi.org/10.1177/0049124101029003005

\section{Publisher's Note}

Springer Nature remains neutral with regard to jurisdictional claims in published maps and institutional affiliations.

Ready to submit your research? Choose BMC and benefit from:

- fast, convenient online submission

- thorough peer review by experienced researchers in your field

- rapid publication on acceptance

- support for research data, including large and complex data types

- gold Open Access which fosters wider collaboration and increased citations

- maximum visibility for your research: over $100 \mathrm{M}$ website views per year

At $\mathrm{BMC}$, research is always in progress.

Learn more biomedcentral.com/submissions 